\title{
Research on the Diffusion Law of Oncomelania Along with the Flow Through a Side-Weir
}

\author{
A.M. Liu* \\ School of Transportation, Wuhan University of Technology, Wuhan, Hubei, 430060, P.R. China
}

\begin{abstract}
Schistosomiasis is a parasitic disease mostly found in areas along the Changjiang River. Oncomelania is the only intermediate host of schistosomiasis. Oncomelania entering into farmland or the residential zones through the flood discharge or irrigation system is the main reason of spreading schistosomiasis. Therefore it is most essential and effective way to control diffusion of oncomelanias along with flow for preventing epidemic of schistosomiasis disease. In order to simulate the flood discharge when the river dike breaks or the flow from main to branch channel, a side-weir flow system is set up in this paper, And further the flow characteristic is studied by experiment and mathematical modeling. Finally the research on the diffusion law of oncomelania along with side-weir flow is carried out using the experimental test. The results show that oncomelanias do not equally distribute at floodplain areas, but place where flow velocity is small, i.e. in the vortex areas during oncomelanias moving along with side-weir flow. The settling positions of oncomelanias are obtained, which lay a good foundation for eliminating oncomelanias.
\end{abstract}

Keywords: Oncomelania, Side-weir flow, Schistosoma, flood discharge, river dike breaks, diffusion law.

\section{INTRODUCTION}

Schistosomiasis is a natural focus infection disease, which is very much influenced by natural factors. Oncomelania is the only intermediate host of schistosomiasis. So it is most essential and effective way to control diffusion of oncomelanias for preventing epidemic of schistosomiasis disease. Since the establishment of new China, it was due to that exterminating oncomelania is set as the basic policy of schistosomiasis japonica prevention, this epidemic was controlled admirably once. But since the $80 \mathrm{~s}$ of the $20^{\text {th }}$ century, epidemic disease situation began to deteriorate. One of the important reasons is that the oncomelanias spread to dyke-enclosed places or the floodplain areas during flood discharge or irrigation [1-3]. This area is heavily populated so that the situation of the spread of schistosomiasis was more and more serious. Common method used to eliminate oncomelanias such as molluscicide, is impossible to be used over a wide area considering its adverse effects on the economic and environment. If the poisonous molluscicidal drugs such as Niclosamide and the PCP-Na are sprinkled in wide area, the water body in living areas are sure to be polluted seriously. On the other hand, people expend a large amount of manpower, material resources and financial resources.

Therefore it is very important to understand the diffusion law of oncomelanias during flood discharge and irrigation for preventing epidemic of schistosomiasis disease. Since the 1980 s a number of researchers investigated the diffusion law

*Address correspondence to this author at School of Transportation, Wuhan University of Technology, Wuhan, Hubei, 430060, P.R. China;

Tel: +86 27 86544877; E-mail: aiming_liu@126.com of oncomelanias along with the flow by adopting the hydrodynamics method. For example, Li Damei [2] explored the sinking behavior and sensitivity to flowing water velocity of oncomelanias in a circular open channel, Zhang Wei etc. Zhang et al. discussed the settling velocity and incipient velocity by the experiment test in a straight flume [4-6]. These research results provide important theories for hydraulic schistosomiasis control project, but the flow characteristics of flood discharge or irrigation is complicated, the diffusion law of oncomelanias in water is not demonstrated clearly. So it's essential to probe the movement and behavior of oncomelanias during the flood discharge or irrigation.

In this paper a side-weir flow system is set up and its flow characteristic is studied by experiment and numerical simulating, the diffusion of oncomelania along with the sideweir flow is discussed. The oncomelanias distribute characteristic is obtained, which provide a useful reference for eliminating oncomelanias more effectively.

\section{EXPERIMENTAL MODEL}

The Circulating flume system with side-weir flow is designed and made so as to model the flow from main to branch channel or from outside dyke-enclosed places to inside these areas during flood discharge. The system arrangement is shown in Figs. (1-3). The system comprises three parts: a flume, a tank and a reservoir. The flume has a length of 16 meters, a width of 0.5 meters and a height of $0.5 \mathrm{~m}$, the side wall of the flume is made of Plexiglas. A standard right-angled triangle weir is set up at the right side of the flume, the lowest points of the weir is $0.2 \mathrm{~m}$ distant from the bottom of the flume. The bottom of the flume is 


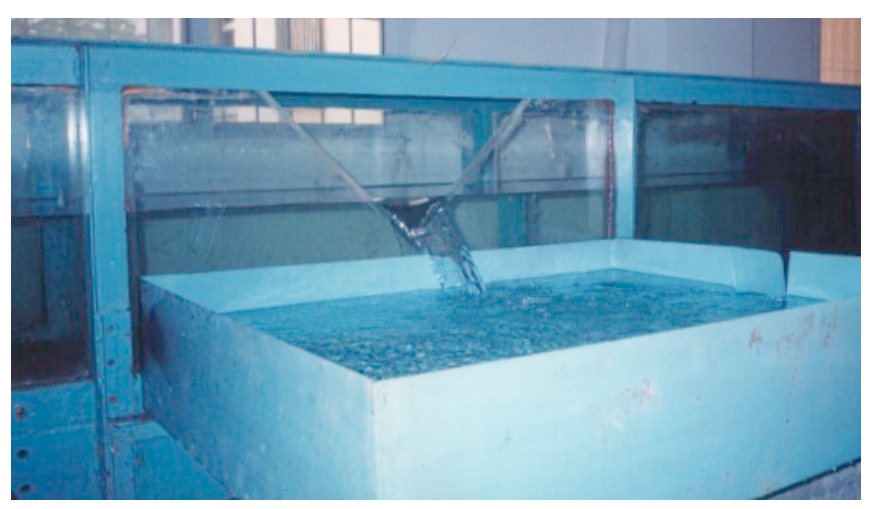

Fig. (1). Experiment scene of side-weir flow.

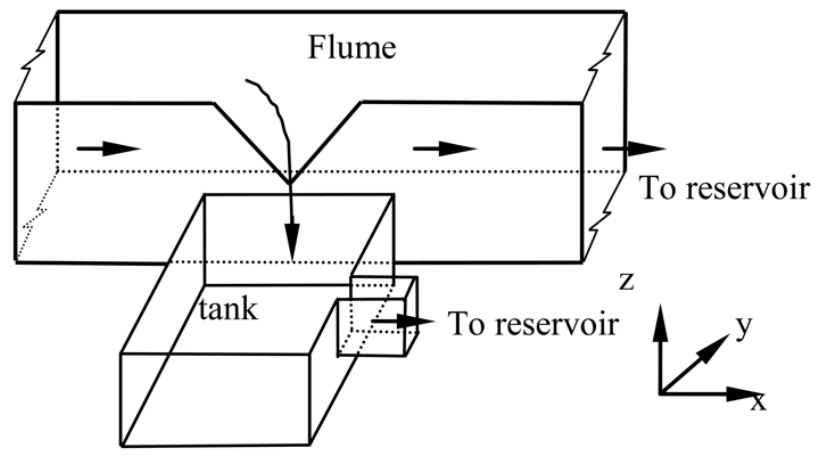

Fig. (2). Diagram of experiment on side weir flow.

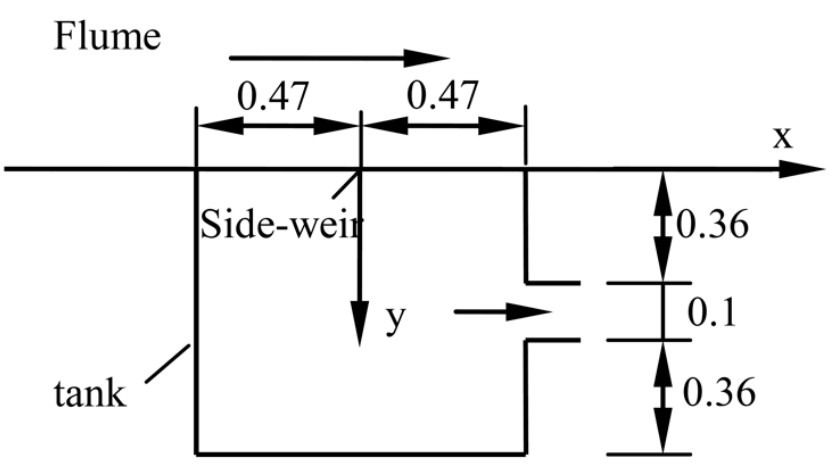

Fig. (3). Planar diagram of experiment on side weir flow (m).

smoothed with cement and its slope is fixed. Upstream flow is divided two parts, one part releases to the reservoir, the other part rushes through the side-weir to the tank, and finally flows from the outlet to the reservoir as well. Thinplate weir is installed at the flume upstream to regulate discharge, and flap gate is used to adjust the stage at the flume downstream as well. The tank is $0.94 \mathrm{~m}$ long and $0.82 \mathrm{~m}$ wide. The outlet is in the middle of the tank's sidewall. The outlet is about rectangular, $0.12 \mathrm{~m}$ long and $0.1 \mathrm{~m}$ wide, $0.07 \mathrm{~m}$ distant from the bottom of the tank.

\section{RESEARCH ON THE FLOW FIELD CHARACTERISTIC}

\subsection{Experimental Testing}

In this paper the water depth of the flume is measured by a water level tracker system. The measuring accuracy is $0.005 \mathrm{~mm}$.
The flow field velocity is measured by Acoustic-Doppler Velocimeter (ADV) as well. The ADV measuring range of velocity is $0.01 \mathrm{~m} / \mathrm{s} \sim 2.5 \mathrm{~m} / \mathrm{s}$ and the accuracy is $\pm 1 \% \mathrm{FS}$. ADV is erected above the flume, and is fixed by a buggy which can move along with longitudinal move of the flume. Step motor on the buggy which can control the vertical and transverse move of the ADV. X, y and $\mathrm{z}$ represent respectively longitudinal, vertical and transverse directions of the channel. The three-dimensional velocity of the flume and the tank is measured. Because the current turns to the aerated water flow when it falls to the tank, it is impossible to measure the velocity in this region by ADV. So the flow is simulated by numerical method as well.

\subsection{Mathematical Model}

Because the flow is complex 3-D turbulent flow with free surface, so it is simulated by double equations model and the free surface is tracked by VOF [7, 8].

According to the VOF method, the free surface is considered interface between air and water. And a volume of fraction $\alpha_{w}$ is introduced to define the water region. In a computational cell, where $\alpha_{w}=1$ in the liquid phase and $\alpha_{w}=0$ in the air phase. Therefore, the air-liquid interface exists in the cell where $\alpha_{w}$ lies between 0 and 1. $\alpha_{w}$ is given by the following equation [8]:

$\frac{\partial \alpha_{w}}{\partial t}+u_{i} \frac{\partial \alpha_{w}}{\partial x_{i}}=0$

So the density of mixture is different from the one phase flow, and is defined as follows:

$\rho=\alpha_{w} \rho_{w}+\left(1-\alpha_{w}\right) \rho_{a}$

$\mu=\alpha_{w} \mu_{w}+\left(1-\alpha_{w}\right) \mu_{a}$

Where $\rho_{a}$ and $\mu_{a}$ is the density and viscosity of air; $\rho_{w}$ and $\mu_{w}$ is the density and viscosity of water.

The governing equation for the flow of the fluids are almost similar to one phase flow except the volume fraction equation when the VOF method is used to track the free surface. These equations are written as follows:

The continuity equation:

$\frac{\partial \rho}{\partial t}+\frac{\partial \rho u_{i}}{\partial x_{i}}=0$

The momentum equation:

$\frac{\partial \rho u_{i}}{\partial t}+\frac{\partial}{\partial x_{j}}\left(\rho u_{i} u_{j}\right)=-\frac{\partial p}{\partial x_{i}}+\frac{\partial}{\partial x_{j}}\left[\left(\mu+\mu_{t}\right)\left(\frac{\partial u_{i}}{\partial x_{j}}+\frac{\partial u_{j}}{\partial x_{i}}\right)\right]$

The equation of turbulence kinetic energy $k$ :

$\left.\frac{\partial \rho k}{\partial t}+\frac{\partial}{\partial x_{j}}\left(\rho u_{i} k\right)=\frac{\partial}{\partial x_{i}}\left[\left(\mu+\frac{\mu_{t}}{\sigma_{k}}\right) \frac{\partial k}{\partial x_{i}}\right)\right]+G+\rho \varepsilon$

The equation of turbulence dissipation ratio $\rho_{w}$ :

$\left.\frac{\partial \rho \varepsilon}{\partial t}+\frac{\partial}{\partial x_{j}}\left(\rho u_{i} \varepsilon\right)=\frac{\partial}{\partial x_{i}}\left[\left(\mu+\frac{\mu_{t}}{\sigma_{\varepsilon}}\right) \frac{\partial \varepsilon}{\partial x_{i}}\right)\right]+C_{1 \varepsilon} \frac{\varepsilon}{k} G-C_{2 \varepsilon} \rho \frac{\varepsilon^{2}}{k}$ 
Where $\mu_{t}$ is turbulent viscosity coefficient that is obtained by turbulence kinetic energy $k$ and turbulence dissipation ratio $\mathcal{E}$ :

$\mu_{t}=\rho C_{\mu} \frac{k^{2}}{\varepsilon}$

Where $c_{\mu}=0.09, \sigma_{k}$ and $\sigma_{\varepsilon}$ are turbulent Prandtl constant of $k$ and $\varepsilon$ respectively, $\sigma_{k}=1.0, \sigma_{\varepsilon}=1.3$.

Where $c_{1 \varepsilon}$ and $c_{2 \varepsilon}$ are $\mathcal{E}$ equation constant, $c_{1 \varepsilon}=1.44$, $c_{2 \varepsilon}=1.92$.

$\mathrm{G}$ is the production term of turbulence kinetic energy due to mean velocity gradient, defined as follows:

$G=\mu_{t}\left(\frac{\partial u_{i}}{\partial x_{j}}+\frac{\partial u_{j}}{\partial x_{i}}\right) \frac{\partial u_{i}}{\partial x_{j}}$

The second order up-wind differencing scheme is used to solve the momentum equation for the sake of minimizing numerical diffusion [8]. The pressure-implicit with splitting of operators (PISO) is adopted for the pressure-velocity coupling scheme [9]. Using PISO allows for a rapid rate of convergence without any significant loss of accuracy.

\subsection{Analysis of the Experimental Test and Numerical Simulation Results}

Two cases of different distributaries discharge through side-weir are researched. The incoming discharge and water depth at the flume upstream are respectively: $Q_{1}=41.68 \mathrm{~L} / \mathrm{s}$, $\mathrm{h}_{1}=25.2 \mathrm{~cm} ; Q_{2}=41.68 L / s, \mathrm{~h}_{2}=28.8 \mathrm{~cm}$.

The water depth of longitudinal section $(5 \mathrm{~cm}$ away from right bank of the flume) is listed in Figs. (4 and 5). From the figures we can see that the computational results accord with the experimental data well, which indicates that the simulation results are reliable.

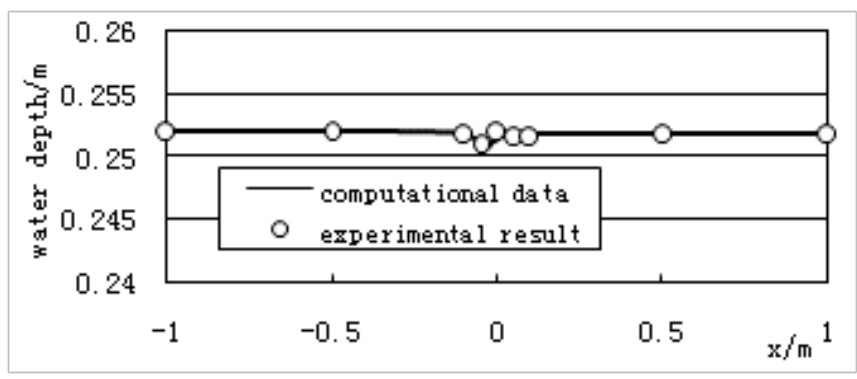

Fig. (4). The water depth of longitudinal section $(5 \mathrm{~cm}$ away from right bank of the flume) for case 1 .

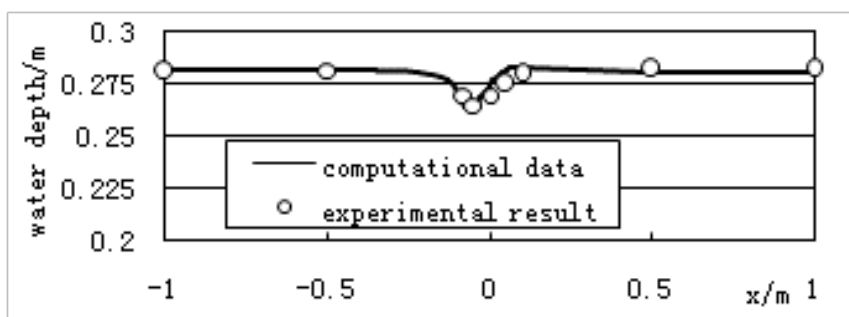

Fig. (5). The water depth of longitudinal section $(5 \mathrm{~cm}$ away from right bank of the flume) for case 1 .
Experimental and computed velocities of the plane at $\mathrm{h}=1 \mathrm{~cm}$ and $\mathrm{h}=4 \mathrm{~cm}$ are shown in Figs. (6-9). The flow characteristic is concluded as follows:

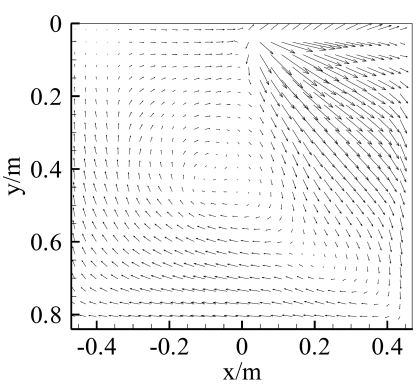

Computational data

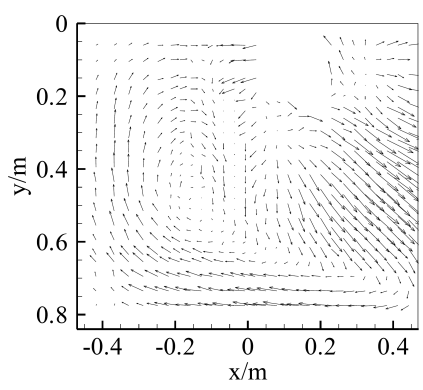

Experimental result
Fig. (6). Computational and experimental velocities of the plane at $\mathrm{h}=1 \mathrm{~cm}$ for case 1 .

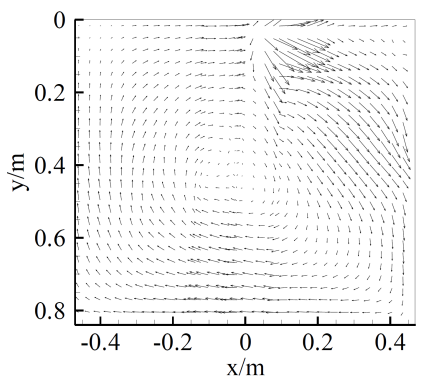

Computational data

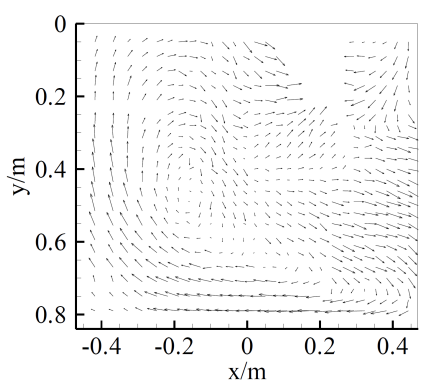

Experimental result
Fig. (7). Computational and experimental velocities of the plane at $\mathrm{h}=4 \mathrm{~cm}$ for case 1 .

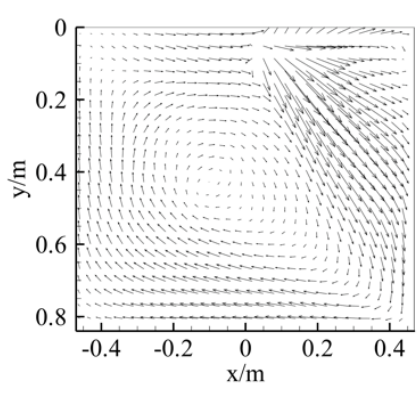

Computational data

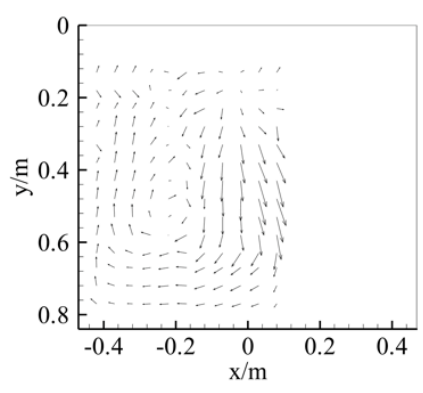

Experimental result
Fig. (8). Computational and experimental velocities of the plane at $\mathrm{h}=1 \mathrm{~cm}$ for case 2 .

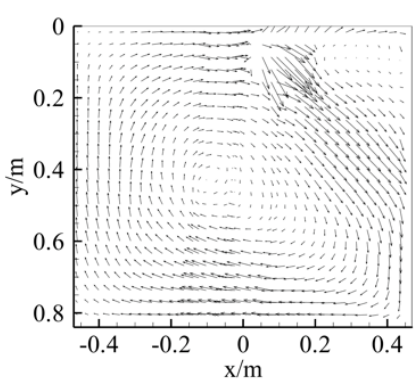

Computational data

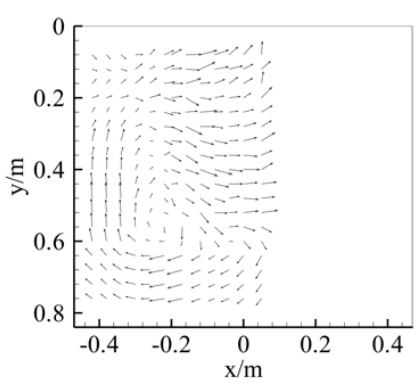

Experimental result
Fig. (9). Computational and experimental velocities of the plane at $\mathrm{h}=4 \mathrm{~cm}$ for case 2 .

(1) The computed velocity is in agreement with the experimental result. Regardless of the discharge through 
the side-weir, there is an obvious vortex in the tank and its scale is similar with that of the tank. As for the same distributary from the flume, the two-dimensional velocity distribution at different water depth plane is similar too. However, the position of the vortex center is changed slightly, that's to say, the closer to the free surface where is, the nearer to the tank downstream the vortex center is. When the current falls to the tank through the side-weir, the flow has a great amount of movement energy and the water changes to aerated flow, so that it is impossible to measure the velocity of the areas by ADV. Especially when the discharge through the side-weir increases, the effective area where the velocity can be measured decreases. Thus we can measure velocity just in the partial area for case 2 .

(2) The position of the vortex center is changed with discharge through the side-weir, i.e., the larger the discharge is, the nearer to the upstream the vortex center is. In addition, the velocity downstream is larger than that in other areas.

(3) There are some difference between calculated and measured values of the position of the vortex center, which may be due to the following reasons: the bottom of the tank is in such a depression but it is considered a flat bottom during the numerical calculation; Secondly the side-weir is thin-plate weir, but the thickness of the weir wall is $5 \mathrm{~mm}$ during the numerical calculation.

\section{THE DIFFUSION LAW OF THE ONCOMELANIA WITH THE FLOW}

On the basis of study on the flow field characteristics, the experimental research on the spread of the oncomelanias with the flow. The oncomelanias used in the experiment are negative, i.e., they are not affected by schistosomiasis. Average water temperature is about $15-18$ degrees. In every test 100 oncomelanias are thrown into the water flume upstream. And we observe the spread characteristics of the oncomelanias with the flow through the side-weir and their transportation in the tank. The spread path of the oncomelanias with the flow and the final sinking position is shown in Fig. (10) and Fig. (11).

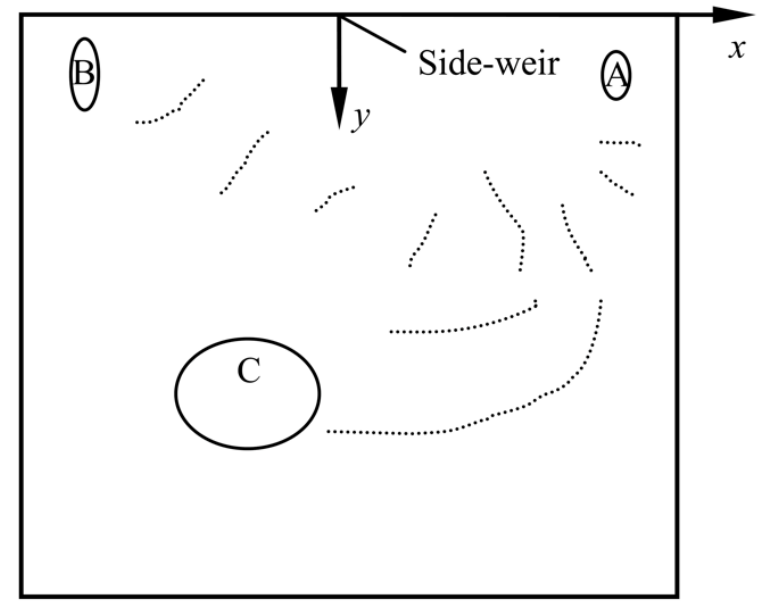

Fig. (10). Spread path of oncomelania along with flow.
From the observation results during the test we can see that the characteristics of the oncomelanias movement with the flow through the side-weir and their transportation in the tank are similar in the two cases.

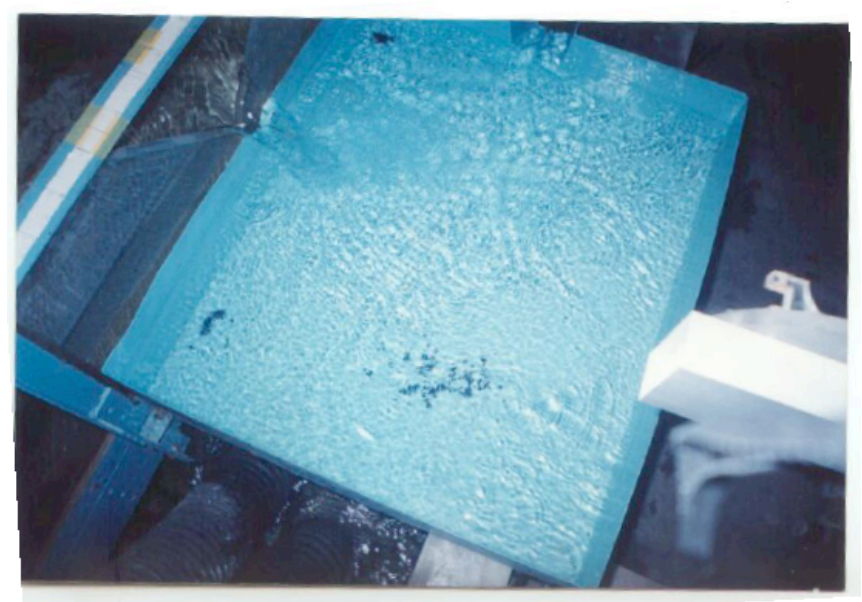

Fig. (11). Sinking position of oncomelanias at the bottom of tank for case 1 .

(1) Because water goes rushing through the side-weir, the oncomelania close its operculum in order to protect themselves, thus these oncomelanias turn to be the state of 'runaway' and move with the flow like sediment particle. Finally they are deposited at the bottom of the tank. After the end of the experiment, the oncomelanias begin to open their operculum and crawl around in the tank.

(2) After water goes rushing through the side-weir, the oncomelanias move quickly upstream, downstream and in the front of the side-weir at the bottom of the tank. A small portion of oncomelanias are settled in region $\mathrm{A}$ and $\mathrm{B}$ near the corner of the tank. Region A is the narrowest, that's to say here the oncomelanias are gathered in a very small area by the flow and show in the shape of 'point distribution'. In regional B distribution of oncomelanias is slightly looser than that in region $\mathrm{A}$. The other portion of oncomelanias move to region $\mathrm{C}$ and stay there finally. In region $\mathrm{C}$ distribution of oncomelanias is slightly looser than that in regions A and B. They show in the shape of 'piece distribution'. It's noticed that most of oncomelanias 'move and wait' and sometimes they are floating during their transportation. $\mathrm{X}$ and $\mathrm{y}$ coordinate values of regions $\mathrm{A}, \mathrm{B}$ and $\mathrm{C}$ center position are shown in Table 1. From Table 1 we can see that in these two conditions distribution locations at the bottom of the tank of the oncomelanias are slightly different.

(3) During the test, no oncomelanias spread to the reservoir through the outlet of the tank.

In summary, the diffusion and distribution of the oncomelanias is in conformity with flow velocity. With the water from the flume falling to the tank, the oncomelanias move quickly forward by the current. However, when they are close to the front-wall of the tank, they are moving and waiting, floating from time to time. Finally they stay at the 
Table 1. $\mathrm{X}$ and $\mathrm{y}$ coordinate values of region $\mathrm{A}, \mathrm{B}$ and $\mathrm{C}$ center position.

\begin{tabular}{|c|c|c|c|c|}
\hline \multirow{2}{*}{$\begin{array}{l}X \text { and } y \text { coordinate values of settling regions and } \\
\text { vortex center }(m)\end{array}$} & \multicolumn{2}{|c|}{ Case 1} & \multicolumn{2}{|c|}{ Case 2} \\
\hline & $\mathbf{x}$ & $\mathbf{y}$ & $\mathbf{x}$ & $\mathbf{y}$ \\
\hline Region B & -0.37 & 0.05 & -0.40 & 0.09 \\
\hline Experimental Vortex center & -0.15 & 0.45 & -0.20 & 0.50 \\
\hline
\end{tabular}

center of the vortex. Because the velocity there is so small that the flow is weak and unable to gather oncomelanias together anymore, distribution of oncomelanias is scattered. In addition, in the corner of the tank in regions $\mathrm{A}$ and $\mathrm{B}$ recirculation is shaped too, where the velocity is small thus a small portion of oncomelanias deposit here. Moreover, with the increase of flow through the side-weir, the vortex center in region $\mathrm{C}$ is closer to upstream, so the oncomelanias move more closer to the upstream.

\section{CONCLUSION}

From the above research results the conclusions about the diffusion law of oncomelanias with the flow can be drawn:

(1) Oncomelanias don't distribute equally throughout the flow field, but distribute in the shape of " point or piece " at the bottom of the tank.

The diffusion and distribution of the oncomelanias is in conformity with that of flow velocity. The majority of oncomelanias deposit in the area of vortex center, a small portion of oncomelanias stay in the area close to the tank corner where the velocity is so small. That's to say after oncomelanias spread by the flow from main channel to branch channel or from outside dyke-enclosed places to inside this areas during discharge and irrigation, they don't distribute evenly throughout the dyke-enclosed places or floodplain area, but stay in the areas where the velocity is so small such as near the bank or vortex center etc.

(2) No oncomelanias spread to the reservoir through the outlet of the tank.

Oncomelania's diffusion law provides an important theory for eliminating oncomelanias.

Common methods used to eliminate oncomelanias such as molluscicide is simple and easily operated, but impossible to be used over a wide area considering its adverse effects on the economy and environment. If people understand the diffusion law of oncomelania along with the flow, drugs are only thrown in the local areas where oncomelanias settle, it will reduce drug pollution to a great extent, and decrease burden on manpower and finance correspondingly. During the test, no oncomelanias spread through the outlet of the tank to reservoir. which also provides the inspiration for active control of flow field for preventing snails to spread with the flow.

Besides author would like to point out there are so many influencing factors on oncomelania activities considering it as a life. Furthermore, natural environment of the floodplain areas or dyke-enclosed places is also very complex, the diffusion law of oncomelania along with the flow is needed to study further.

\section{CONFLICT OF INTEREST}

The author confirms that this article content has no conflict of interest.

\section{ACKNOWLEDGEMENTS}

This work was supported by CRSRI Open Research Program (CKWV2013200/KY) and National Natural Science Foundation of China (51309182)

\section{REFERENCES}

[1] C. Li, "Advances in research on prevention of snails spreading through culvert and sluice", Express Water Resour. Hydropow. Info., Vol. 5, pp. 23-26, 1998 (in Chinese).

[2] D. Li, "Study of water intakes for oncomelania control based on information behavior and CFD results", Sci. China, Series E Technolog. Sci., Vol. 44, pp. 522-530, 2001.

[3] D. Li, Oncomelania area of Three Gorges Project Downstream Change Tendency and Counter measure. Wuhan, China, 1997, pp. 24-42 (in Chinese).

[4] W. Zhang, Z. Xong, X. Yang and X. Xu, "Study on settling velocity of snails in flowing water and flume experiment", Yangtze River Scientif. Res. Inst., Vol. 11, pp. 62-68, 1994 (in Chinese).

[5] W. Zhang and Z. Xong, "Experimental study on flow velocity of incipient snail motion", Yangtze River Scientif. Res. Inst., Vol. 11, pp. 24-29, 1994 (in Chinese).

[6] X. Yang, X. Xu, J. Liu and Y. Mao, "Experimental research on subsidence and motion pattern of snails in current water", Ch. J. Schistosom. Control, Vol. 6, pp. 137-139, 1994 (in Chinese).

[7] C. W. Hirt and B. D. Nichols, "Volume of fluid (VOF) methods for the dynamics of free boundaries", J. Computat. Phys., Vol. 39, pp. 201-225, 1981. 
[8] D. L. Youngs, Time-Dependent Multi-Material flow with Large fluid Distortion, In: K. W. Morton and M. J. Baines, Eds. Numerical Methods for Fluid Dynamics, Academic Press, New York, 1982, pp. 273-285.
[9] R. I. Issa, "Solution of implicity discretized fluid flow equations by operator splitting", J. Computat. Phys., Vol. 62, pp. 40-65, 1986.

(C) A.M. Liu; Licensee Bentham Open.

This is an open access article licensed under the terms of the Creative Commons Attribution Non-Commercial License (http://creativecommons.org/licenses/ by-nc/4.0/) which permits unrestricted, non-commercial use, distribution and reproduction in any medium, provided the work is properly cited. 